\title{
Robust $L_{2}-L_{\infty}$ Control of Uncertain Differential Linear Repetitive Processes
}

\author{
Ligang $\mathrm{Wu}$ and Zidong Wang*
}

\begin{abstract}
For two-dimensional (2-D) systems, information propagates in two independent directions. 2-D systems are known to have both system-theoretic and applications interest, and the so-called linear repetitive processes (LRPs) are a distinct class of 2-D discrete linear systems. This paper is concerned with the problem of $L_{2}-L_{\infty}$ (energy to peak) control for uncertain differential LRPs, where the parameter uncertainties are assumed to be norm-bounded. For an unstable LRP, our attention is focused on the design of an $L_{2}-L_{\infty}$ static state feedback controller and an $L_{2}-L_{\infty}$ dynamic output feedback controller, both of which guarantee the corresponding closed-loop LRPs to be stable along the pass and have a prescribed $L_{2}-L_{\infty}$ performance. Sufficient conditions for the existence of such $L_{2}-L_{\infty}$ controllers are proposed in terms of linear matrix inequalities (LMIs). The desired $L_{2}-L_{\infty}$ dynamic output feedback controller can be found by solving a convex optimization problem. A numerical example is provided to demonstrate the effectiveness of the proposed controller design procedures.
\end{abstract}

\section{Keywords}

Dynamic output feedback control, linear matrix inequality (LMI), linear repetitive processes (LRPs), $L_{2}-L_{\infty}$ performance, uncertainty

\section{INTRODUCTION}

Many practical systems can be modeled as two-dimensional (2-D) systems, such as those in image data processing and transmission, thermal processes, gas absorption and water stream heating [12]. Therefore, in recent years much attention has been devoted to the analysis and synthesis problems for 2-D systems, and many important results have been easily available in the literature. To mention a few, the stability problem of 2-D systems has been investigated in [1], the controller and filter design problems have been studied in [1,24], and the model approximation problem for 2-D systems has been addressed in [5]. Linear repetitive processes (LRPs), on the other hand, are a distinct class of 2-D linear systems with applications in areas ranging from long-wall coal cutting through to iterative learning control schemes [17]. A special feature of LRPs is that information propagation in one of the two distinct directions only occurs over a finite duration. The essentially unique characteristic of a repetitive (or multipass) process is a series of sweeps, termed passes, through a set of dynamics defined over a fixed finite duration known as the pass length. On each pass, an output, termed the pass profile, is produced which acts as a forcing function on, and hence contributes to, the dynamics of the next pass profile [17]. Recently, differential LRPs have received much attention and many valuable results on designing the control law towards stability and performance purposes have been reported in the literature, see for example $[3,15]$ and references therein. The guaranteed cost controller for its uncertain case has been designed in [14] and, very recently, the $H_{\infty}$ control problem has been studied in [15].

This work was supported in part by the Engineering and Physical Sciences Research Council (EPSRC) of the U.K. under Grant GR/S27658/01, the Nuffield Foundation of the U.K. under Grant NAL/00630/G, and the Alexander von Humboldt Foundation of Germany.

L. Wu is with the College of Automation Science and Engineering, South China University of Technology, Guangzhou, 510641, P. R. China. Email: ligangwu@hit.edu.cn

Z. Wang is with the Department of Information Systems and Computing, Brunel University, Uxbridge, Middlesex, UB8 3PH, United Kingdom.

${ }^{*}$ Corresponding author. Email: Zidong. Wang@brunel.ac.uk 
In designing controllers for LRPs, one could apply a linear combination of the current pass state vector and the previous pass profile, based on the assumption that the process state is completely accessible to feedback. such an assumption, however, is not always valid in practice since some state components cannot be measured. There are two commonly used methods to deal with the controller design problem in the case that the process state components are not accessible. One is to design a state observer in order to estimate the immeasurable state components and then synthesize an observer-based controller, and the other is to design a feedback controller by using the measurable output information. The latter is usually classified into static or dynamic output feedback control [10]. Generally speaking, dynamic output feedback is more flexible than static output feedback since additional dynamics of the controller is introduced. Although dynamic output feedback involves more design parameters, for linear systems, the closed-loop system can usually be written as in a more compact form where certain parameters can be embedded into augmented matrix variables. Such a compact formulation, as shown in [15], can be conveniently converted into a convex optimization problem using linear matrix inequalities (LMIs) that can be effectively solved with numerical optimization packages.

There are basically two complementary approaches to the design of LMI-based output feedback controllers, that is, the well-known variable elimination procedure and the linearizing variables transform method. The linearizing variables transform method introduces a general framework to formulate a synthesis problem as a convex optimization one involving LMIs, where the main idea is to apply specific invertible transforms of the controller parameters in order to achieve LMI conditions by means of a new set of variables. When the resulted LMIs are solvable, the controller parameters can be computed by applying the inverse transforms. This approach might lose computational effectiveness when the number of decision variables grows up. In such a case, an elimination of some decision variables may still be required which, unfortunately, can only be applied on specific structures of the underlying matrix inequalities. It should be noted that existing results on designing an $H_{\infty}$ dynamic pass profile controller have only been based on the variables elimination method, see e.g. [16]. Hence, there is a natural need to provide an alternative design method by using the linearizing variables transform method, especially for systems of low dimensions.

On the other hand, over the past decades, a powerful robust control framework has been developed for addressing issues of stability and performance in the presence of uncertainties. Robust stability and performance are achieved by minimizing an appropriate norm (such as $\|\cdot\|_{L_{2}-L_{2}}\left(\right.$ or $\left.\|\cdot\|_{\infty}\right),\|\cdot\|_{L_{2}-L_{\infty}}$ and $\|\cdot\|_{L_{\infty}-L_{\infty}}$, which are called energy to energy, energy to peak, and peak to peak, respectively) of a transfer function. Considerable attention has been devoted to the optimal control problem, such as energy to energy (or $H_{\infty}$ ) control and model reduction $[4,6,25,26], H_{2}$ (and mixed $H_{2} / H_{\infty}$ ) control and filtering $[8,9,19,20,22,23]$, energy to peak (or $L_{2}-L_{\infty}$ ) control and filtering [7,21]. In many practical cases, the $L_{2}-L_{\infty}$ performance is more reasonable to achieve in system design. Note that $L_{2}-L_{\infty}$ control design has received considerable research attention mainly because of its insensitivity to the exact knowledge of the statistics of the noise signals. Such a control procedure ensures that the $L_{2}-L_{\infty}$ gain from the noise input signals to the controlled output will be less than a prescribed level, where the noise input is an arbitrary energy-bounded signal. Since LRPs have wide applications in areas ranging from long-wall coal cutting through to iterative learning control schemes, a controlled LPR with guaranteed $L_{2}-L_{\infty}$ performance would have more robustness against exogenous disturbances, and is therefore more important in practical controller design especially presented with an uncertain environment. Several methods have been proposed to solve the $L_{2}-L_{\infty}$ control, see, for example, [13,21] and the references therein. However, to date, little work has been reported on $L_{2}-L_{\infty}$ controller design for differential LRPs, despite the practical motivation as well as the great importance.

In this paper, we are interested in using the $L_{2}-L_{\infty}$ norm as the optimization objective in the controller synthesis that minimizes the worst case amplification from disturbance signal to controlled output signal, where the disturbance signal size (norm) is taken to be energy value and the controlled output signal size is taken to be peak value. Linear uncertain differential LRPs are considered, where the parameter uncertainties 
are assumed to be norm-bounded. For an unstable LRP, we aim to design an $L_{2}$ - $L_{\infty}$ static state feedback controller and an $L_{2}-L_{\infty}$ dynamic output feedback controller such that the corresponding closed-loop LRPs are stable along the pass and a prescribed $L_{2}-L_{\infty}$ performance is guaranteed. It is shown that the desired $L_{2}-L_{\infty}$ dynamic output feedback controller can be found by solving a convex optimization problem using standard numerical software [2]. A numerical example is provided to demonstrate the effectiveness of the proposed controller design procedures.

The rest of this paper is organized as follows. The $L_{2}-L_{\infty}$ control problem for uncertain differential LRPs is formulated in Section 2. Section 3 presents our main results. An illustrative example is provided in Section 4 and we conclude this paper in Section 5.

Notations. The notations used throughout the paper are standard. The superscript " $T$ " stands for matrix transposition; $\mathbb{R}^{n}$ denotes the $n$-dimensional Euclidean space, $\mathbb{R}^{m \times n}$ is the set of all real matrices of dimension $m \times n$ and the notation $P>0$ means that $P$ is real symmetric and positive definite; $I$ and 0 represent identity matrix and zero matrix; $|\cdot|$ refers to the Euclidean vector norm; and $\lambda_{\min }(\cdot), \lambda_{\max }(\cdot)$ denote the minimum and the maximum eigenvalues of a real symmetric matrix, respectively. In symmetric block matrices or long matrix expressions, we use an asterisk $*$ to represent a term that is induced by symmetry, $\operatorname{diag}(.$.$) stands for$ a block-diagonal matrix and $\operatorname{sym}(A)$ denotes $A+A^{T}$. Matrices, if their dimensions are not explicitly stated, are assumed to be compatible for algebraic operations.

\section{Process Description and Problem Formulation}

The uncertain differential LRPs considered here are described in a state-space model of the following form over $0 \leq t \leq \alpha$ (where $\alpha$ is an integer denoting the pass length) and $k \geq 0$ :

$$
\begin{aligned}
\Sigma: \quad \dot{x}_{k+1}(t) & =(A+\Delta A) x_{k+1}(t)+\left(B_{0}+\Delta B_{0}\right) y_{k}(t)+(B+\Delta B) u_{k+1}(t)+B_{1} \omega_{k+1}(t) \\
y_{k+1}(t) & =(C+\Delta C) x_{k+1}(t)+\left(D_{0}+\Delta D_{0}\right) y_{k}(t)+(D+\Delta D) u_{k+1}(t)+D_{1} \omega_{k+1}(t)
\end{aligned}
$$

where, on pass $k, x_{k}(t) \in \mathbb{R}^{n}$ is the state vector; $y_{k}(t) \in \mathbb{R}^{m}$ is the pass profile vector; $u_{k}(t) \in \mathbb{R}^{q}$ is the control input; $\omega_{k}(t) \in \mathbb{R}^{l}$ is the disturbance input which belongs to $L_{2}\{[0, \infty),[0, \infty)\} ; A, B_{0}, B, B_{1}, C, D_{0}$, $D$ and $D_{1}$ are real constant matrices; $\Delta A, \Delta B_{0}, \Delta B, \Delta C, \Delta D_{0}$ and $\Delta D$ are parameter uncertainties which are norm-bounded and can be described by

$$
\left[\begin{array}{ccc}
\Delta A & \Delta B_{0} & \Delta B \\
\Delta C & \Delta D_{0} & \Delta D
\end{array}\right]=\left[\begin{array}{l}
M_{1} \\
M_{2}
\end{array}\right] \Delta(t)\left[\begin{array}{lll}
N_{1} & N_{2} & N_{3}
\end{array}\right],
$$

where $M_{1}, M_{2}, N_{1}, N_{2}$ and $N_{3}$ are real constant matrices, and $\Delta(t)$ is a real uncertain matrix function with Lebesgue measurable elements satisfying $\Delta^{T}(t) \Delta(t) \leq I$. To complete the process description, it is necessary to specify the boundary conditions for the state initial vector on each pass and the initial pass profile (that is, on pass 0):

$$
\begin{aligned}
x_{k+1}(0) & =d_{k+1}, \quad \forall k \geq 0, \\
y_{0}(t) & =f(t),
\end{aligned}
$$

where $d_{k+1} \in \mathbb{R}^{n}$ is known constant entries and $f(t) \in \mathbb{R}^{m}$ is an vector whose entries are known functions of $t$ over $[0, \alpha]$.

Consider a static feedback controller with the following general structure:

$$
\hat{\Sigma}_{S}: \quad u_{k+1}(t)=\left[\begin{array}{ll}
K_{1} & K_{2}
\end{array}\right]\left[\begin{array}{c}
x_{k+1}(t) \\
y_{k}(t)
\end{array}\right]
$$


where $K_{1}$ and $K_{2}$ are appropriately dimensioned matrices to be designed. Then, the resulting closed-loop process can be formulated as

$$
\begin{aligned}
\tilde{\Sigma}_{S}: \quad \dot{x}_{k+1}(t) & =(\hat{A}+\Delta \hat{A}) x_{k+1}(t)+\left(\hat{B}_{0}+\Delta \hat{B}_{0}\right) y_{k}(t)+B_{1} \omega_{k+1}(t) \\
y_{k+1}(t) & =(\hat{C}+\Delta \hat{C}) x_{k+1}(t)+\left(\hat{D}_{0}+\Delta \hat{D}_{0}\right) y_{k}(t)+D_{1} \omega_{k+1}(t)
\end{aligned}
$$

where

$$
\begin{aligned}
\hat{A} & \triangleq A+B K_{1}, \quad \hat{B}_{0} \triangleq B_{0}+B K_{2}, \quad \hat{C} \triangleq C+D K_{1}, \quad \hat{D}_{0} \triangleq D_{0}+D K_{2}, \\
\Delta \hat{A} & \triangleq \Delta A+\Delta B K_{1}, \quad \Delta \hat{B}_{0} \triangleq \Delta B_{0}+\Delta B K_{2}, \quad \Delta \hat{C} \triangleq \Delta C+\Delta D K_{1}, \quad \Delta \hat{D}_{0} \triangleq \Delta D_{0}+\Delta D K_{2}
\end{aligned}
$$

with

$$
\left[\begin{array}{cc}
\Delta \hat{A} & \Delta \hat{B}_{0} \\
\Delta \hat{C} & \Delta \hat{D}_{0}
\end{array}\right]=\left[\begin{array}{c}
M_{1} \\
M_{2}
\end{array}\right] \Delta(t)\left[\begin{array}{cc}
N_{1}+N_{3} K_{1} & N_{2}+N_{3} K_{2}
\end{array}\right] .
$$

The above addressed static feedback controller requires the current pass state $x_{k+1}(t)$ and the pass profile $y_{k}(t)$ to be fully accessible, which is very restrictive in practical applications. In such a case, one option is to assume the availability of a so-called measured output signal vector given by

$$
z_{k+1}(t)=L x_{k+1}(t)+F_{0} y_{k}(t)+F_{1} \omega_{k+1}(t)
$$

where $z_{k}(t) \in \mathbb{R}^{r}$ is the measured output, $L, F_{0}$ and $F_{1}$ are real constant matrices. The controlled output signal is given by

$$
v_{k+1}(t)=G x_{k+1}(t)+H_{0} y_{k}(t)
$$

where $v_{k}(t) \in \mathbb{R}^{q}, G$ and $H_{0}$ are real constant matrices.

Here, we are also interested in designing a full-order dynamic output feedback controller of general structure described by $\hat{\Sigma}_{D}$ :

$$
\begin{aligned}
\hat{\Sigma}_{D}: \quad \dot{\varphi}_{k+1}(t) & =A_{c} \varphi_{k+1}(t)+B_{0 c} \phi_{k}(t)+B_{c} z_{k+1}(t) \\
\phi_{k+1}(t) & =C_{c} \varphi_{k+1}(t)+D_{0 c} \phi_{k}(t)+D_{c} z_{k+1}(t) \\
u_{k+1}(t) & =G_{c} \varphi_{k+1}(t)+H_{0 c} \phi_{k}(t)+H_{c} z_{k+1}(t)
\end{aligned}
$$

where, on pass $k, \varphi_{k}(t) \in \mathbb{R}^{n}$ is the controller state vector, $\phi_{k}(t) \in \mathbb{R}^{m}$ is the pass profile vector of controller, and $A_{c}, B_{0 c}, B_{c}, C_{c}, D_{0 c}, D_{c}, G_{c}, H_{0 c}$ and $H_{c}$ are appropriately dimensioned constant matrices to be determined.

Now, augmenting the model of $\Sigma$ to include the state of controller $\hat{\Sigma}_{D}$, we obtain the following closed-loop process $\tilde{\Sigma}_{D}$ :

$$
\begin{aligned}
\tilde{\Sigma}_{D}: \quad \xi_{k+1}(t) & =(\tilde{A}+\Delta \tilde{A}) \xi_{k+1}(t)+\left(\tilde{B}_{0}+\Delta \tilde{B}_{0}\right) \zeta_{k}(t)+\left(\tilde{B}_{1}+\Delta \tilde{B}_{1}\right) \omega_{k+1}(t) \\
\zeta_{k+1}(t) & =(\tilde{C}+\Delta \tilde{C}) \xi_{k+1}(t)+\left(\tilde{D}_{0}+\Delta \tilde{D}_{0}\right) \zeta_{k}(t)+\left(\tilde{D}_{1}+\Delta \tilde{D}_{1}\right) \omega_{k+1}(t) \\
v_{k+1}(t) & =\tilde{G} \xi_{k+1}(t)+\tilde{H}_{0} \zeta_{k}(t)
\end{aligned}
$$

where $\xi_{k+1}(t) \triangleq\left[\begin{array}{ll}x_{k+1}^{T}(t) & \varphi_{k+1}^{T}(t)\end{array}\right]^{T}, \zeta_{k}(t) \triangleq\left[\begin{array}{cc}y_{k}^{T}(t) & \phi_{k}^{T}(t)\end{array}\right]^{T}$ and

$$
\left[\begin{array}{ccc}
\Delta \tilde{A} & \Delta \tilde{B}_{0} & \Delta \tilde{B}_{1} \\
\Delta \tilde{C} & \Delta \tilde{D}_{0} & \Delta \tilde{D}_{1}
\end{array}\right] \triangleq\left[\begin{array}{c}
\tilde{M}_{1} \\
\tilde{M}_{2}
\end{array}\right] \Delta(t)\left[\begin{array}{ccc}
\tilde{N}_{1} & \tilde{N}_{2} & \tilde{N}_{3}
\end{array}\right]
$$


with

$$
\begin{aligned}
& \tilde{A} \triangleq\left[\begin{array}{cc}
A+B H_{c} L & B G_{c} \\
B_{c} L & A_{c}
\end{array}\right], \tilde{B}_{0} \triangleq\left[\begin{array}{cc}
B_{0}+B H_{c} F_{0} & B H_{0 c} \\
B_{c} F_{0} & B_{0 c}
\end{array}\right], \tilde{B}_{1} \triangleq\left[\begin{array}{c}
B_{1}+B H_{c} F_{1} \\
B_{c} F_{1}
\end{array}\right], \\
& \tilde{C} \triangleq\left[\begin{array}{cc}
C+D H_{c} L & D G_{c} \\
D_{c} L & C_{c}
\end{array}\right], \tilde{D}_{0} \triangleq\left[\begin{array}{cc}
D_{0}+D H_{c} F_{0} & D H_{0 c} \\
D_{c} F_{0} & D_{0 c}
\end{array}\right], \tilde{D}_{1} \triangleq\left[\begin{array}{c}
D_{1}+D H_{c} F_{1} \\
D_{c} F_{1}
\end{array}\right], \\
& \tilde{G} \triangleq\left[\begin{array}{cc}
G & 0
\end{array}\right], \tilde{H}_{0} \triangleq\left[\begin{array}{cc}
H_{0} & 0
\end{array}\right], \tilde{N}_{1} \triangleq\left[\begin{array}{cc}
N_{1}+N_{3} H_{c} L & N_{3} G_{c}
\end{array}\right], \tilde{N}_{3} \triangleq N_{3} H_{c} F_{1}, \\
& \tilde{M}_{1} \triangleq\left[\begin{array}{c}
M_{1} \\
0
\end{array}\right], \tilde{M}_{2} \triangleq\left[\begin{array}{c}
M_{2} \\
0
\end{array}\right], \tilde{N}_{2} \triangleq\left[\begin{array}{cc}
N_{2}+N_{3} H_{c} F_{0} & N_{3} H_{0 c}
\end{array}\right] .
\end{aligned}
$$

Before formulating the problems of this section, we give the following definition.

Definition 1: The differential LRP in (1) is said to have an $L_{2}-L_{\infty}$ performance, if it is robustly stable along the pass and, under zero boundary conditions and for all nonzero $\omega_{k+1}(t) \in L_{2}\{[0, \infty),[0, \infty)\}$, the following inequality

$$
\left\|y_{k+1}(t)\right\|_{\infty, \alpha}<\gamma_{2, \infty}\left\|\omega_{k+1}(t)\right\|_{2, \alpha} \quad\left(\gamma_{2, \infty}>0\right)
$$

holds, where $\gamma_{2, \infty}>0$ is a given real scalar and the corresponding norms are define by

$$
\left\|f_{k}(t)\right\|_{2, \alpha} \triangleq \sqrt{\sum_{k=0}^{\infty} \int_{0}^{\alpha} f_{k}^{T}(t) f_{k}(t) d t}, \quad\left\|f_{k}(t)\right\|_{\infty, \alpha} \triangleq \sqrt{\sup _{k \geq 0, t \in[0, \alpha]} f_{k}^{T}(t) f_{k}(t)}
$$

The problems to be investigated in this paper can be formulated as:

- Static Feedback Control. Design a static feedback controller in (4) such that the closed-loop differential LRP in (5) is robustly stable along the pass and has the specified $L_{2}-L_{\infty}$ performance, that is, satisfies (14).

- Dynamic Output Feedback Control. Design a dynamic output feedback controller in (10) such that the closed-loop differential LRP in (11) is robustly stable along the pass and has the specified $L_{2}-L_{\infty}$ performance, that is, satisfies

$$
\left\|v_{k+1}(t)\right\|_{\infty, \alpha}<\gamma_{2, \infty}\left\|\omega_{k+1}(t)\right\|_{2, \alpha} \quad\left(\gamma_{2, \infty}>0\right)
$$

We end this section by giving two lemmas which will be used in the sequel.

Lemma 1: [11] Let $\Sigma_{1}, \Sigma_{2}$ be real matrices of appropriate dimensions. Then, for any matrix $\Delta$ satisfying $\Delta^{T} \Delta \leq I$ and a scalar $\epsilon>0$, the following inequality holds:

$$
\Sigma_{1} \Delta \Sigma_{2}+\Sigma_{2}^{T} \Delta^{T} \Sigma_{1}^{T} \leq \epsilon^{-1} \Sigma_{1} \Sigma_{1}^{T}+\epsilon \Sigma_{2}^{T} \Sigma_{2}
$$

Lemma 2: [3] The nominal differential LRP (1) with $u_{k+1}(t)=0$ and $\omega_{k+1}(t)=0$ is stable along the pass if there exist matrices $P_{1}>0$ and $P_{2}>0$ such that the following LMI is feasible:

$$
\left[\begin{array}{ccc}
P_{1} A+A^{T} P_{1} & P_{1} B_{0} & C^{T} P_{2} \\
* & -P_{2} & D_{0}^{T} P_{2} \\
* & * & -P_{2}
\end{array}\right]<0
$$

\section{MAIN RESUlTS}

\section{A. Robust $L_{2}-L_{\infty}$ performance analysis}

In this subsection, we shall analyze the robust stability along the pass as well as the $L_{2}-L_{\infty}$ performance for the uncertain differential LRP in (1) with $u_{k+1}(t)=0$.

Theorem 1: For a given scalar $\gamma_{2, \infty}>0$, the uncertain differential LRP in (1) with $u_{k+1}(t)=0$ is robustly stable along the pass and has an $L_{2}-L_{\infty}$ performance level $\gamma_{2, \infty}$ (i.e., (14) is satisfied), if there exist matrices 
$P_{1}>0, P_{2}>0$ and scalars $\epsilon_{1}>0, \epsilon_{2}>0$ such that the following LMIs are feasible:

Proof:

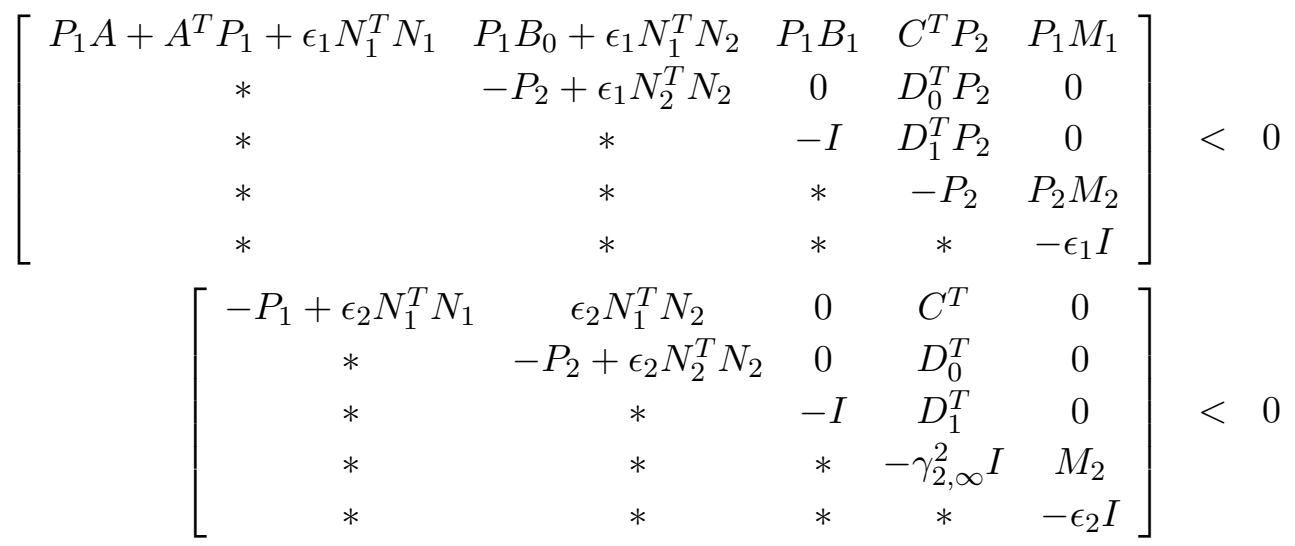

First, we establish the stability along the pass of the nominal process $\Sigma$ in $(1)$ with $\omega_{k+1}(t)=0$. Choose the following Lyapunov functional candidate:

$$
\begin{aligned}
V(k, t) & \triangleq V_{1}(t ; k)+V_{2}(k ; t), \\
V_{1}(t ; k) & \triangleq x_{k+1}^{T}(t) P_{1} x_{k+1}(t), \\
V_{2}(k ; t) & \triangleq y_{k}^{T}(t) P_{2} y_{k}(t)
\end{aligned}
$$

where $P_{1}>0$ and $P_{2}>0$ are matrices to be found. Consider the increment $\Delta V(k, t)$ given by

$$
\Delta V(k, t) \triangleq \frac{\partial V_{1}(t ; k)}{\partial t}+\Delta V_{2}(k ; t)
$$

and define

$$
\sum_{k=0}^{\infty} \int_{0}^{\alpha} \Delta V(k, t) d t \triangleq \int_{0}^{\alpha} \frac{\partial V_{1}(t ; k)}{\partial t} d t+\sum_{k=0}^{\infty} \Delta V_{2}(k ; t)
$$

Then, along the solution of the nominal process $\Sigma$, we have

$$
\begin{aligned}
\frac{\partial V_{1}(t ; k)}{\partial t} & =2 x_{k+1}^{T}(t) P_{1} \dot{x}_{k+1}(t)=2 x_{k+1}^{T}(t) P_{1}\left[A x_{k+1}(t)+B_{0} y_{k}(t)\right] \\
\Delta V_{2}(k ; t) & =y_{k+1}^{T}(t) P_{2} y_{k+1}(t)-y_{k}^{T}(t) P_{2} y_{k}(t) \\
& =\left[C x_{k+1}(t)+D_{0} y_{k}(t)\right]^{T} P_{2}\left[C x_{k+1}(t)+D_{0} y_{k}(t)\right]-y_{k}^{T}(t) P_{2} y_{k}(t)
\end{aligned}
$$

It follows that

$$
\begin{aligned}
\Delta V(k, t)= & 2 x_{k+1}^{T}(t) P_{1}\left[A x_{k+1}(t)+B_{0} y_{k}(t)\right]+\left[C x_{k+1}(t)+D_{0} y_{k}(t)\right]^{T} \\
& \times P_{2}\left[C x_{k+1}(t)+D_{0} y_{k}(t)\right]-y_{k}^{T}(t) P_{2} y_{k}(t) \\
= & \varsigma_{k}^{T}(t)\left(\check{P}_{1} \check{A}+\check{A}^{T} \check{P}_{1}+\check{C}^{T} \check{P}_{2} \check{C}-\check{P}_{2}\right) \varsigma_{k}(t) \triangleq \varsigma_{k}^{T}(t) \Psi \varsigma_{k}(t),
\end{aligned}
$$

where $\Psi \triangleq \check{P}_{1} \check{A}+\check{A}^{T} \check{P}_{1}+\check{C}^{T} \check{P}_{2} \check{C}-\check{P}_{2}$ and

$$
\varsigma_{k}(t) \triangleq\left[\begin{array}{c}
x_{k+1}(t) \\
y_{k}(t)
\end{array}\right], \check{A} \triangleq\left[\begin{array}{cc}
A & B_{0} \\
0 & 0
\end{array}\right], \check{C} \triangleq\left[\begin{array}{cc}
0 & 0 \\
C & D_{0}
\end{array}\right], \check{P}_{1} \triangleq\left[\begin{array}{cc}
P_{1} & 0 \\
0 & 0
\end{array}\right], \check{P}_{2} \triangleq\left[\begin{array}{cc}
0 & 0 \\
0 & P_{2}
\end{array}\right] .
$$

Since LMI (18) implies $\Psi<0$, for any $\varsigma_{k}(t) \neq 0$, we have $\Delta V(k, t)<0$ which indicates the stability along the pass of the nominal process $\Sigma$ in (1) with $\omega_{k+1}(t)=0$. 
Next, in order to establish the $L_{2}-L_{\infty}$ performance, we assume the zero boundary conditions, that is, $x_{k+1}(0)=0(\forall k \geq 0)$ and $y_{0}(t)=0$. Consider the following performance index:

$$
\mathcal{I}=V(k, t)-\sum_{s=0}^{k-1} \int_{0}^{t} \omega_{s+1}^{T}(\beta) \omega_{s+1}(\beta) d \beta .
$$

According to the stability along the pass of the process and the zero boundary conditions, we have

$$
\begin{aligned}
\mathcal{I} & =V(k, t)-V_{1}(0 ; k)-V_{2}(0 ; t)-\sum_{s=0}^{k-1} \int_{0}^{t} \omega_{s+1}^{T}(\beta) \omega_{s+1}(\beta) d \beta \\
& =\int_{0}^{t} \frac{\partial V_{1}(\beta ; k)}{\partial \beta} d \beta+\sum_{s=0}^{k-1} \Delta V_{2}(s ; t)-\sum_{s=0}^{k-1} \int_{0}^{t} \omega_{s+1}^{T}(\beta) \omega_{s+1}(\beta) d \beta \\
& =\sum_{s=0}^{k-1} \int_{0}^{t}\left[\Delta V(s, \beta)-\omega_{s+1}^{T}(\beta) \omega_{s+1}(\beta)\right] d \beta \\
& \triangleq \sum_{s=0}^{k-1} \int_{0}^{t} \eta_{s}^{T}(\beta) \Omega \eta_{s}(\beta) d \beta
\end{aligned}
$$

where

$$
\eta_{s}(\beta) \triangleq\left[\begin{array}{c}
x_{s+1}(\beta) \\
y_{s}(\beta) \\
\omega_{s+1}(\beta)
\end{array}\right], \quad \Omega \triangleq\left[\begin{array}{ccc}
P_{1} A+A^{T} P_{1} & P_{1} B_{0} & P_{1} B_{1} \\
* & -P_{2} & 0 \\
* & * & -I
\end{array}\right]+\left[\begin{array}{c}
C^{T} \\
D_{0}^{T} \\
D_{1}^{T}
\end{array}\right] P_{2}\left[\begin{array}{c}
C^{T} \\
D_{0}^{T} \\
D_{1}^{T}
\end{array}\right]^{T}
$$

Notice that if

$$
\left[\begin{array}{cccc}
P_{1} A+A^{T} P_{1} & P_{1} B_{0} & P_{1} B_{1} & C^{T} P_{2} \\
* & -P_{2} & 0 & D_{0}^{T} P_{2} \\
* & * & -I & D_{1}^{T} P_{2} \\
* & * & * & -P_{2}
\end{array}\right]<0,
$$

we have $\Omega<0$ by Schur complement, and therefore for all $\eta_{k}(p) \neq 0$ we have $\mathcal{I}<0$, i.e.

$$
x_{k+1}^{T}(t) P_{1} x_{k+1}(t)+y_{k}^{T}(t) P_{2} y_{k}(t)=V(k, t)<\sum_{s=0}^{k-1} \int_{0}^{t} \omega_{s+1}^{T}(\beta) \omega_{s+1}(\beta) d \beta .
$$

On the other hand, LMI (19) implies

$$
\left[\begin{array}{cccc}
-P_{1} & 0 & 0 & C^{T} \\
* & -P_{2} & 0 & D_{0}^{T} \\
* & * & -I & D_{1}^{T} \\
* & * & * & -\gamma_{2, \infty}^{2} I
\end{array}\right]<0
$$

that is,

$$
\left[\begin{array}{c}
C^{T} \\
D_{0}^{T} \\
D_{1}^{T}
\end{array}\right]\left[\begin{array}{lll}
C & D_{0} & D_{1}
\end{array}\right]<\gamma_{2, \infty}^{2}\left[\begin{array}{ccc}
P_{1} & 0 & 0 \\
0 & P_{2} & 0 \\
0 & 0 & I
\end{array}\right]
$$


Therefore, we can conclude from the nominal process (1) and (29)-(31) that, for any $k>0$ and $t \in[0, \alpha]$, the following holds:

$$
\begin{aligned}
y_{k+1}^{T}(t) y_{k+1}(t) & =\left[C x_{k+1}(t)+D_{0} y_{k}(t)+D_{1} \omega_{k+1}(t)\right]^{T}\left[C x_{k+1}(t)+D_{0} y_{k}(t)+D_{1} \omega_{k+1}(t)\right] \\
& <\gamma_{2, \infty}^{2}\left[x_{k+1}^{T}(t) P_{1} x_{k+1}(t)+y_{k}^{T}(t) P_{2} y_{k}(t)+\omega_{k+1}^{T}(t) \omega_{k+1}(t)\right] \\
& <\gamma_{2, \infty}^{2}\left[\sum_{s=0}^{k-1} \int_{0}^{t} \omega_{s+1}^{T}(\beta) \omega_{s+1}(\beta) d \beta+\omega_{k+1}^{T}(t) \omega_{k+1}(t)\right] \\
& <\gamma_{2, \infty}^{2} \sum_{s=0}^{\infty} \int_{0}^{\alpha} \omega_{s+1}^{T}(\beta) \omega_{s+1}(\beta) d \beta .
\end{aligned}
$$

Taking the supremum over $k>0$ and $t \in[0, \alpha]$ yields $\left\|y_{k+1}(t)\right\|_{\infty, \alpha}<\gamma_{2, \infty}\left\|\omega_{k+1}(t)\right\|_{2, \alpha}$.

Now, let us consider the parameter uncertainties. By replacing $A, B_{0}, C$ and $D_{0}$ with $(A+\Delta A),\left(B_{0}+\Delta B_{0}\right)$, $(C+\Delta C)$ and $\left(D_{0}+\Delta D_{0}\right)$, respectively, we can see that the uncertain differential LRP in (1) with $u_{k+1}(t)=0$ is robustly stable along the pass and has an $L_{2}-L_{\infty}$ performance, if there exist matrices $P_{1}>0, P_{2}>0$ satisfying

$$
\begin{gathered}
{\left[\begin{array}{cccc}
P_{1} A+A^{T} P_{1} & P_{1} B_{0} & P_{1} B_{1} & C^{T} P_{2} \\
* & -P_{2} & 0 & D_{0}^{T} P_{2} \\
* & * & -I & D_{1}^{T} P_{2} \\
* & * & * & -P_{2}
\end{array}\right]+\operatorname{sym}\left(\left[\begin{array}{c}
P_{1} M_{1} \\
0 \\
0 \\
P_{2} M_{2}
\end{array}\right] \Delta\left[\begin{array}{c}
N_{1}^{T} \\
N_{2}^{T} \\
0 \\
0
\end{array}\right]\right) \quad\left(\left[\begin{array}{c}
0 \\
0 \\
0 \\
M_{2}
\end{array}\right] \Delta\left[\begin{array}{cccc}
-P_{1} & 0 & 0 & C^{T} \\
* & -P_{2} & 0 & D_{0}^{T} \\
* & * & -I & D_{1}^{T} \\
* & * & * & -\gamma_{2, \infty}^{2} I
\end{array}\right]+\operatorname{sym}\left(\begin{array}{c}
N_{1}^{T} \\
N_{2}^{T} \\
0 \\
0
\end{array}\right]\right)<0}
\end{gathered}
$$

Furthermore, by invoking Lemma 1 together with Schur complement, (33) and (34) hold if (18) and (19) hold, and then the proof is completed.

\section{B. Static $L_{2}-L_{\infty}$ control}

We are in a position to present a solution to the $L_{2}-L_{\infty}$ static feedback control problem.

Theorem 2: Consider the uncertain differential LRP in (1), and let $\gamma_{2, \infty}>0$ be a prescribed scalar. There exists a feedback controller of the form (4) such that the closed-loop LRP in (5) is robustly stable along the pass and has an $L_{2}-L_{\infty}$ performance level $\gamma_{2, \infty}$ (i.e., (14) is satisfied), if there exist matrices $\mathcal{P}_{1}>0, \mathcal{P}_{2}>0$, $\mathcal{X}, \mathcal{Y}$ and scalars $\lambda_{1}>0, \lambda_{2}>0$ such that the following LMIs are feasible:

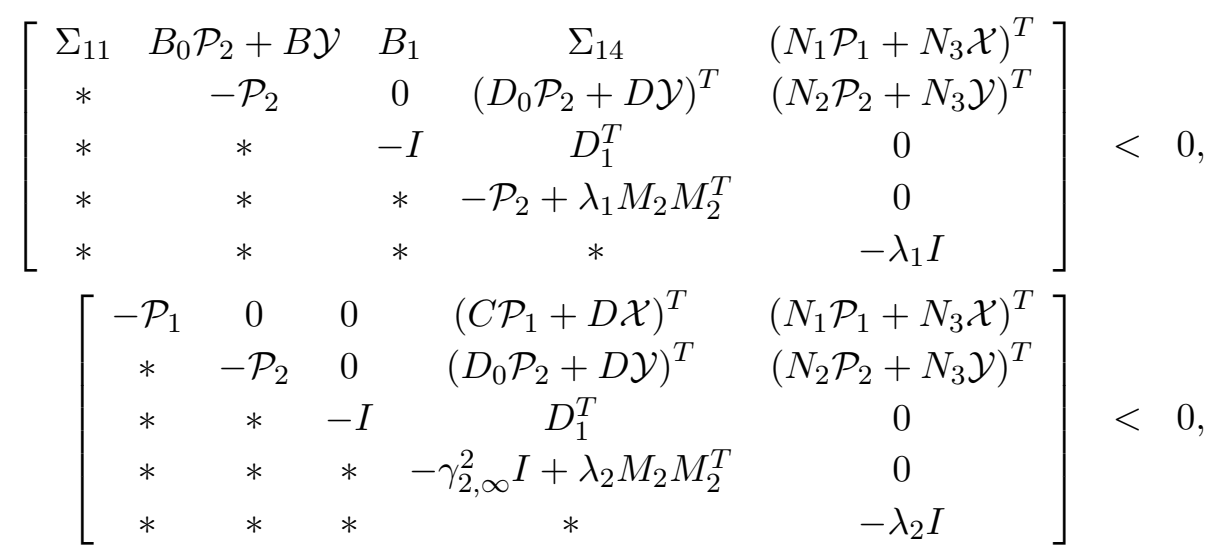


where

$$
\Sigma_{11} \triangleq \operatorname{sym}\left(A \mathcal{P}_{1}+B \mathcal{X}\right)+\lambda_{1} M_{1} M_{1}^{T}, \quad \Sigma_{14} \triangleq\left(C \mathcal{P}_{1}+D \mathcal{X}\right)^{T}+\lambda_{1} M_{1} M_{2}^{T}
$$

Moreover, if these conditions hold, the matrices $K_{1}$ and $K_{2}$ are given by $\mathcal{X} \mathcal{P}_{1}^{-1}$ and $\mathcal{Y} \mathcal{P}_{2}^{-1}$, respectively.

Proof: Substituting the controller (4) into (1), we obtain the closed-loop process as in (5). According to the proof of Theorem 1, replacing $A, B_{0}, C, D_{0}, \Delta A, \Delta B_{0}, \Delta C$ and $\Delta D_{0}$ with $\hat{A}, \hat{B}_{0}, \hat{C}, \hat{D}_{0}, \Delta \hat{A}, \Delta \hat{B}_{0}$, $\Delta \hat{C}$ and $\Delta \hat{D}_{0}$ in (6)-(7), respectively, we can see that the closed-loop LRP in (5) is robustly stable along the pass and has an $L_{2}-L_{\infty}$ performance, if there exist matrices $P_{1}>0, P_{2}>0$ satisfying

$$
\begin{aligned}
& {\left[\begin{array}{cccc}
P_{1}\left(A+B K_{1}\right)+\left(A+B K_{1}\right)^{T} P_{1} & P_{1}\left(B_{0}+B K_{2}\right) & P_{1} B_{1} & \left(C+D K_{1}\right)^{T} P_{2} \\
* & -P_{2} & 0 & \left(D_{0}+D K_{2}\right)^{T} P_{2} \\
* & * & -I & D_{1}^{T} P_{2} \\
* & * & * & -P_{2}
\end{array}\right]} \\
& +\operatorname{sym}\left(\left[\begin{array}{c}
P_{1} M_{1} \\
0 \\
0 \\
P_{2} M_{2}
\end{array}\right] \Delta\left[\begin{array}{c}
\left(N_{1}+N_{3} K_{1}\right)^{T} \\
\left(N_{2}+N_{3} K_{2}\right)^{T} \\
0 \\
0
\end{array}\right]^{T}\right)<0 \\
& {\left[\begin{array}{cccc}
-P_{1} & 0 & 0 & \left(C+D K_{1}\right)^{T} \\
* & -P_{2} & 0 & \left(D_{0}+D K_{2}\right)^{T} \\
* & * & -I & D_{1}^{T} \\
* & * & * & -\gamma_{2, \infty}^{2} I
\end{array}\right]+\operatorname{sym}\left(\left[\begin{array}{c}
0 \\
0 \\
0 \\
M_{2}
\end{array}\right] \Delta\left[\begin{array}{c}
\left(N_{1}+N_{3} K_{1}\right)^{T} \\
\left(N_{2}+N_{3} K_{2}\right)^{T} \\
0 \\
0
\end{array}\right]^{T}\right]^{T}<.}
\end{aligned}
$$

Performing congruence transformations to (37) and (38) by $\operatorname{diag}\left(P_{1}^{-1}, P_{2}^{-1}, I, P_{2}^{-1}\right)$ and $\operatorname{diag}\left(P_{1}^{-1}, P_{2}^{-1}\right.$, $I, I)$, we have

$$
\begin{aligned}
& {\left[\begin{array}{cccc}
\left(A+B K_{1}\right) P_{1}^{-1}+P_{1}^{-1}\left(A+B K_{1}\right)^{T} & \left(B_{0}+B K_{2}\right) P_{2}^{-1} & B_{1} & P_{1}^{-1}\left(C+D K_{1}\right)^{T} \\
* & -P_{2}^{-1} & 0 & P_{2}^{-1}\left(D_{0}+D K_{2}\right)^{T} \\
* & * & -I & D_{1}^{T} \\
* & * & * & -P_{2}^{-1}
\end{array}\right]} \\
& +\operatorname{sym}\left(\left[\begin{array}{c}
M_{1} \\
0 \\
0 \\
M_{2}
\end{array}\right] \Delta\left[\begin{array}{c}
P_{1}^{-1}\left(N_{1}+N_{3} K_{1}\right)^{T} \\
P_{2}^{-1}\left(N_{2}+N_{3} K_{2}\right)^{T} \\
0 \\
0
\end{array}\right]^{T}\right)<0 \\
& {\left[\begin{array}{cccc}
-P_{1}^{-1} & 0 & 0 & P_{1}^{-1}\left(C+D K_{1}\right)^{T} \\
* & -P_{2}^{-1} & 0 & P_{2}^{-1}\left(D_{0}+D K_{2}\right)^{T} \\
* & * & -I & D_{1}^{T} \\
* & * & * & -\gamma_{2, \infty}^{2} I
\end{array}\right]+\operatorname{sym}\left(\left[\begin{array}{c}
0 \\
0 \\
0 \\
M_{2}
\end{array}\right] \Delta\left[\begin{array}{c}
P_{1}^{-1}\left(N_{1}+N_{3} K_{1}\right)^{T} \\
P_{2}^{-1}\left(N_{2}+N_{3} K_{2}\right)^{T} \\
0 \\
0
\end{array}\right]^{T}\right)}
\end{aligned}
$$

Defining $\mathcal{P}_{1}=P_{1}^{-1}, \mathcal{P}_{2}=P_{2}^{-1}, K_{1} \mathcal{P}_{1}=\mathcal{X}, K_{2} \mathcal{P}_{2}=\mathcal{Y}$ and invoking Lemma 1 together with Schur complement, we know that (39) and (40) hold if (35) and (36) hold, and this concludes the proof.

\section{Dynamic $L_{2}-L_{\infty}$ output feedback control}

In this subsection, we shall give the main results of $L_{2}-L_{\infty}$ dynamic output feedback control of uncertain differential linear repetitive processes. First, we state the following theorem without proof, since the proof can be obtained along the same line of reasoning as in the derivation of Theorem 1. 
Theorem 3: For a given scalar $\gamma_{2, \infty}>0$, the closed-loop LRP $\tilde{\Sigma}_{D}$ in (11) is robustly stable along the pass and has an $L_{2}-L_{\infty}$ performance level $\gamma_{2, \infty}$ (i.e., (15) is satisfied), if there exist matrices $P>0$ and $Q>0$ and scalars $\epsilon_{1}>0, \epsilon_{2}>0$ such that the following LMIs hold:

$$
\left.\begin{array}{ccccc}
P \tilde{A}+\tilde{A}^{T} P+\epsilon_{1} \tilde{N}_{1}^{T} \tilde{N}_{1} & P \tilde{B}_{0}+\epsilon_{1} \tilde{N}_{1}^{T} \tilde{N}_{2} & P \tilde{B}_{1}+\epsilon_{1} \tilde{N}_{1}^{T} \tilde{N}_{3} & \tilde{C}^{T} Q & P \tilde{M}_{1} \\
* & -Q+\epsilon_{1} \tilde{N}_{2}^{T} \tilde{N}_{2} & \epsilon_{1} \tilde{N}_{2}^{T} \tilde{N}_{3} & \tilde{D}_{0}^{T} Q & 0 \\
* & * & -I+\epsilon_{1} \tilde{N}_{3}^{T} \tilde{N}_{3} & \tilde{D}_{1}^{T} Q & 0 \\
* & * & * & -Q & Q \tilde{M}_{2} \\
* & * & * & * & -\epsilon_{1} I
\end{array}\right]<\begin{aligned}
& \\
& \\
&
\end{aligned}
$$

where $\tilde{A}, \tilde{B}_{0}, \tilde{B}_{1}, \tilde{C}, \tilde{D}_{0}, \tilde{D}_{1}, \tilde{G}, \tilde{H}_{0}, \tilde{M}_{1}, \tilde{M}_{2}, \tilde{N}_{1}, \tilde{N}_{2}$ and $\tilde{N}_{3}$ are defined in (12)-(13).

Finally, let us present a solution to the $L_{2}-L_{\infty}$ dynamic output feedback control problem.

Theorem 4: Consider the uncertain differential LRP $\Sigma$ in (1) and let $\gamma_{2, \infty}>0$ be a prescribed scalar. There exists a dynamic output feedback controller $\hat{\Sigma}_{D}$ in the form of (10) such that the closed-loop LRP $\tilde{\Sigma}_{D}$ in (11) is robustly stable along the pass and has an $L_{2}-L_{\infty}$ performance level $\gamma_{2, \infty}$ (i.e., (15) is satisfied), if there exist matrices $\mathcal{P}>0, \mathcal{R}>0, \mathcal{Q}>0, \mathcal{S}>0, \mathcal{A}_{c}, \mathcal{B}_{0 c}, \mathcal{B}_{c}, \mathcal{C}_{c}, \mathcal{D}_{0 c}, \mathcal{D}_{c}, \mathcal{G}_{c}, \mathcal{H}_{0 c}, \mathcal{H}_{c}$ and scalars $\lambda_{1}>0, \lambda_{2}>0$ such that the following LMIs hold:

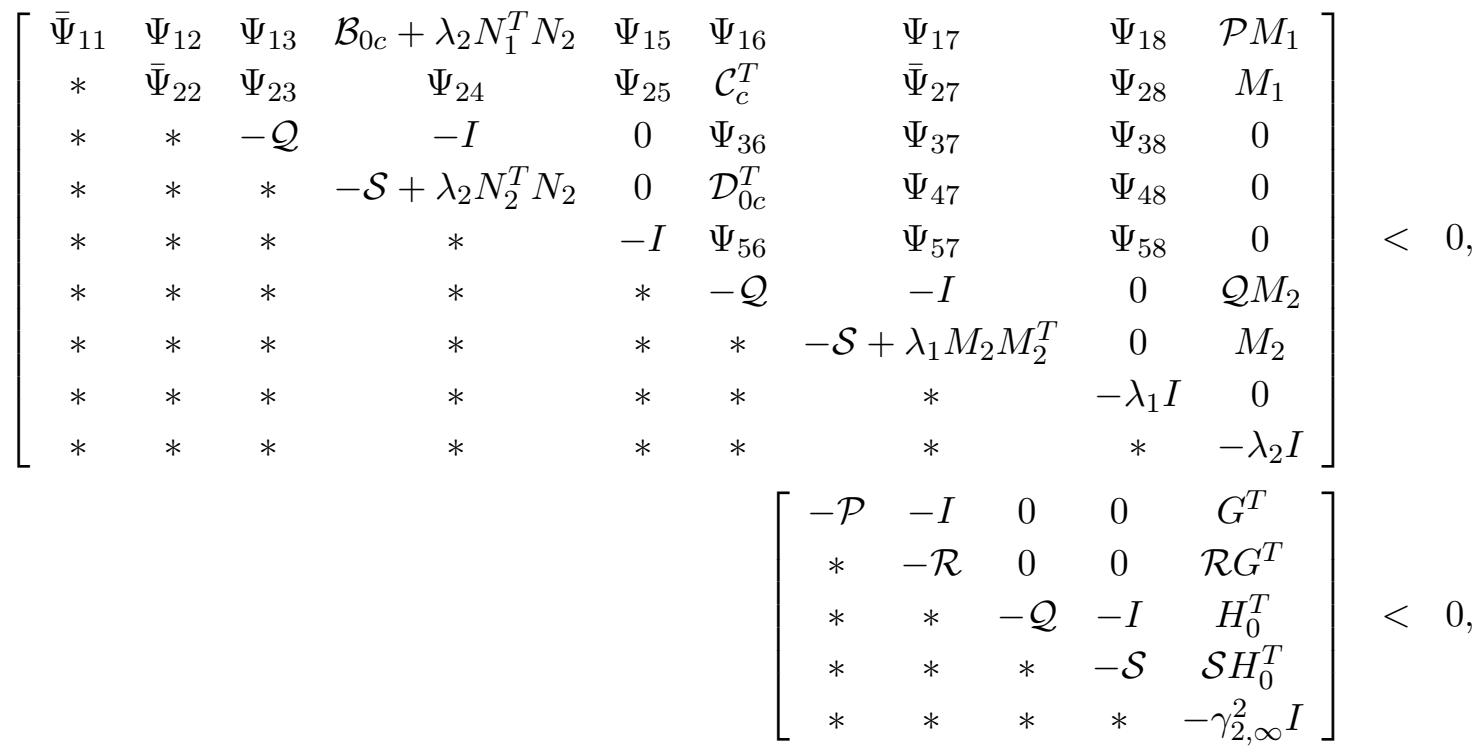

where $\bar{\Psi}_{11} \triangleq \Psi_{11}+\lambda_{2} N_{1}^{T} N_{1}, \bar{\Psi}_{22} \triangleq \Psi_{22}+\lambda_{1} M_{1} M_{1}^{T}, \bar{\Psi}_{27} \triangleq \Psi_{27}+\lambda_{1} M_{1} M_{2}^{T}$ and

$$
\begin{aligned}
& \Psi_{11} \triangleq \operatorname{sym}\left(\mathcal{P} A+\mathcal{B}_{c} L\right), \quad \Psi_{12} \triangleq \mathcal{A}_{c}+\left(A+B \mathcal{H}_{c} L\right)^{T}, \quad \Psi_{13} \triangleq \mathcal{P} B_{0}+\mathcal{B}_{c} F_{0}, \\
& \Psi_{22} \triangleq \operatorname{sym}\left(A \mathcal{R}+B \mathcal{G}_{c}\right), \quad \Psi_{23} \triangleq B_{0}+B \mathcal{H}_{c} F_{0}, \quad \Psi_{24} \triangleq B_{0} \mathcal{S}+B \mathcal{H}_{0 c}, \\
& \Psi_{15} \triangleq \mathcal{P} B_{1}+\mathcal{B}_{c} F_{1}, \quad \Psi_{25} \triangleq B_{1}+B \mathcal{H}_{c} F_{1}, \quad \Psi_{16} \triangleq\left(\mathcal{Q} C+\mathcal{D}_{c} L\right)^{T}, \\
& \Psi_{36} \triangleq\left(\mathcal{Q} D_{0}+\mathcal{D}_{c} F_{0}\right)^{T}, \quad \Psi_{56} \triangleq\left(\mathcal{Q} D_{1}+\mathcal{D}_{c} F_{1}\right)^{T}, \quad \Psi_{17} \triangleq\left(C+D \mathcal{H}_{c} L\right)^{T}, \\
& \Psi_{27} \triangleq\left(C \mathcal{R}+D \mathcal{G}_{c}\right)^{T}, \quad \Psi_{37} \triangleq\left(D_{0}+D \mathcal{H}_{c} F_{0}\right)^{T}, \quad \Psi_{47} \triangleq\left(D_{0} \mathcal{S}+D \mathcal{H}_{0 c}\right)^{T}, \\
& \Psi_{57} \triangleq\left(D_{1}+D \mathcal{H}_{c} F_{1}\right)^{T}, \quad \Psi_{18} \triangleq\left(N_{3} \mathcal{H}_{c} L\right)^{T}, \quad \Psi_{28} \triangleq\left(N_{1} \mathcal{R}+N_{3} \mathcal{G}_{c}\right)^{T}, \\
& \Psi_{38} \triangleq\left(N_{3} \mathcal{H}_{c} F_{0}\right)^{T}, \quad \Psi_{48} \triangleq\left(N_{2} \mathcal{S}+N_{3} \mathcal{H}_{0 c}\right)^{T}, \quad \Psi_{58} \triangleq\left(N_{3} \mathcal{H}_{c} F_{1}\right)^{T} .
\end{aligned}
$$

Moreover, a desired $L_{2}-L_{\infty}$ dynamic output feedback controller in (10) with parameters $\left(A_{c}, B_{0 c}, B_{c}, C_{c}, D_{0 c}\right.$, 
$\left.D_{c}, G_{c}, H_{0 c}, H_{c}\right)$ can be found by solving the following equations:

$$
\left\{\begin{array}{l}
\mathcal{H}_{c} \triangleq H_{c} \\
\mathcal{H}_{0 c} \triangleq H_{c} F_{0} \mathcal{S}+H_{0 c} S_{12}^{T} \\
\mathcal{G}_{c} \triangleq H_{c} L \mathcal{R}+G_{c} R_{12}^{T} \\
\mathcal{D}_{c} \triangleq \mathcal{Q} D H_{c}+Q_{12} D_{c} \\
\mathcal{B}_{c} \triangleq \mathcal{P} B H_{c}+P_{12} B_{c} \\
\mathcal{D}_{0 c} \triangleq \mathcal{Q}\left(D_{0}+D H_{c} F_{0}\right) \mathcal{S}+Q_{12} D_{c} F_{0} \mathcal{S}+\mathcal{Q} D H_{0 c} S_{12}^{T}+Q_{12} D_{0 c} S_{12}^{T} \\
\mathcal{C}_{c} \triangleq \mathcal{Q}\left(C+D H_{c} L\right) \mathcal{R}+Q_{12} D_{c} L \mathcal{R}+\mathcal{Q} D G_{c} R_{12}^{T}+Q_{12} C_{c} R_{12}^{T} \\
\mathcal{B}_{0 c} \triangleq \mathcal{P}\left(B_{0}+B H_{c} F_{0}\right) \mathcal{S}+P_{12} B_{c} F_{0} \mathcal{S}+\mathcal{P} B H_{0 c} S_{12}^{T}+P_{12} B_{0 c} S_{12}^{T} \\
\mathcal{A}_{c} \triangleq \mathcal{P}\left(A+B H_{c} L\right) \mathcal{R}+P_{12} B_{c} L \mathcal{R}+\mathcal{P} B G_{c} R_{12}^{T}+P_{12} A_{c} R_{12}^{T}
\end{array}\right.
$$

Proof: First, we consider the nominal closed-loop LRP $\tilde{\Sigma}_{D}$ in (11). It follows from the proof of Theorem 3 that (11) is stable along the pass and satisfies (15), if there exist matrices $P>0$ and $Q>0$ such that (42) and the following LMI holds:

$$
\left[\begin{array}{cccc}
P \tilde{A}+\tilde{A}^{T} P & P \tilde{B}_{0} & P \tilde{B}_{1} & \tilde{C}^{T} Q \\
* & -Q & 0 & \tilde{D}_{0}^{T} Q \\
* & * & -I & \tilde{D}_{1}^{T} Q \\
* & * & * & -Q
\end{array}\right]<0 .
$$

It can also be seen from Theorem 3 that, since $P>0$ and $Q>0$, the matrices $P$ and $Q$ are nonsingular if (41) holds. By denoting $R=P^{-1} S=Q^{-1}$, we partition $P, R, Q, S$ as follows:

$$
P \triangleq\left[\begin{array}{ll}
P_{11} & P_{12} \\
P_{12}^{T} & P_{22}
\end{array}\right], R=P^{-1} \triangleq\left[\begin{array}{ll}
R_{11} & R_{12} \\
R_{12}^{T} & R_{22}
\end{array}\right], Q \triangleq\left[\begin{array}{cc}
Q_{11} & Q_{12} \\
Q_{12}^{T} & Q_{22}
\end{array}\right], S=Q^{-1} \triangleq\left[\begin{array}{cc}
S_{11} & S_{12} \\
S_{12}^{T} & S_{22}
\end{array}\right]
$$

As we are considering a full-order controller, $P_{12}$ and $R_{12}$ are square. Without loss of generality, we suppose $P_{12}$ and $R_{12}$ are nonsingular [18] (if not, $P_{12}$ and $R_{12}$ may be perturbed by matrices $\Delta P_{12}$ and $\Delta R_{12}$, respectively, with sufficiently small norms such that $P_{12}+\Delta P_{12}$ and $R_{12}+\Delta R_{12}$ are nonsingular and satisfying (43)), with the same principle as above, we also assume that $Q_{12}$ and $Q_{12}$ are nonsingular without loss of generality, and then we can define the following matrices which are also nonsingular:

$$
\Gamma_{P} \triangleq\left[\begin{array}{cc}
P_{11} & I \\
P_{12}^{T} & 0
\end{array}\right], \Gamma_{R} \triangleq\left[\begin{array}{cc}
I & R_{11} \\
0 & R_{12}^{T}
\end{array}\right], \Gamma_{Q} \triangleq\left[\begin{array}{cc}
Q_{11} & I \\
Q_{12}^{T} & 0
\end{array}\right], \Gamma_{S} \triangleq\left[\begin{array}{cc}
I & S_{11} \\
0 & S_{12}^{T}
\end{array}\right]
$$

Note that $P \Gamma_{R}=\Gamma_{P}, Q \Gamma_{S}=\Gamma_{Q}$ and

$$
P_{11} R_{11}+P_{12} R_{12}^{T}=I, \quad Q_{11} S_{11}+Q_{12} S_{12}^{T}=I .
$$

Performing congruence transformations to (47) and (42) by $\operatorname{diag}\left(\Gamma_{R}, \Gamma_{S}, I, \Gamma_{S}\right)$ and $\operatorname{diag}\left(\Gamma_{R}, \Gamma_{S}, I\right)$ respectively, we have

$$
\begin{gathered}
{\left[\begin{array}{cccc}
\Gamma_{P}^{T} \tilde{A} \Gamma_{R}+\Gamma_{R}^{T} \tilde{A}^{T} \Gamma_{P} & \Gamma_{P}^{T} \tilde{B}_{0} \Gamma_{S} & \Gamma_{P}^{T} \tilde{B}_{1} & \Gamma_{R}^{T} \tilde{C}^{T} \Gamma_{Q} \\
* & -\Gamma_{S}^{T} \Gamma_{Q} & 0 & \Gamma_{S}^{T} \tilde{D}_{0}^{T} \Gamma_{Q} \\
* & * & -I & \tilde{D}_{1}^{T} \Gamma_{Q} \\
* & * & * & -\Gamma_{S}^{T} \Gamma_{Q}
\end{array}\right]<0,} \\
\\
{\left[\begin{array}{ccc}
-\Gamma_{P}^{T} \Gamma_{R} & 0 & \Gamma_{R}^{T} \tilde{G}^{T} \\
* & -\Gamma_{Q}^{T} \Gamma_{S} & \Gamma_{S}^{T} \tilde{H}_{0}^{T} \\
* & * & -\gamma_{2, \infty}^{2} I
\end{array}\right]<0 .}
\end{gathered}
$$


Defining $\mathcal{P} \triangleq P_{11}, \mathcal{R} \triangleq R_{11}, \mathcal{Q} \triangleq Q_{11}, \mathcal{S} \triangleq S_{11}$ and the following matrices:

$$
\left\{\begin{array}{l}
\mathcal{A}_{c} \triangleq P_{11}\left(A+B H_{c} L\right) R_{11}+P_{12} B_{c} L R_{11}+P_{11} B G_{c} R_{12}^{T}+P_{12} A_{c} R_{12}^{T} \\
\mathcal{B}_{0 c} \triangleq P_{11}\left(B_{0}+B H_{c} F_{0}\right) S_{11}+P_{12} B_{c} F_{0} S_{11}+P_{11} B H_{0 c} S_{12}^{T}+P_{12} B_{0 c} S_{12}^{T} \\
\mathcal{C}_{c} \triangleq Q_{11}\left(C+D H_{c} L\right) R_{11}+Q_{12} D_{c} L R_{11}+Q_{11} D G_{c} R_{12}^{T}+Q_{12} C_{c} R_{12}^{T} \\
\mathcal{D}_{0 c} \triangleq Q_{11}\left(D_{0}+D H_{c} F_{0}\right) S_{11}+Q_{12} D_{c} F_{0} S_{11}+Q_{11} D H_{0 c} S_{12}^{T}+Q_{12} D_{0 c} S_{12}^{T} \\
\mathcal{B}_{c} \triangleq P_{11} B H_{c}+P_{12} B_{c} \\
\mathcal{D}_{c} \triangleq Q_{11} D H_{c}+Q_{12} D_{c} \\
\mathcal{G}_{c} \triangleq H_{c} L R_{11}+G_{c} R_{12}^{T} \\
\mathcal{H}_{0 c} \triangleq H_{c} F_{0} S_{11}+H_{0 c} S_{12}^{T} \\
\mathcal{H}_{c} \triangleq H_{c}
\end{array}\right.
$$

and considering (12)-(13), we have the following expressions:

$$
\begin{aligned}
& \Gamma_{P}^{T} \tilde{A} \Gamma_{R} \triangleq\left[\begin{array}{cc}
\mathcal{P} A+\mathcal{B}_{c} L & \mathcal{A}_{c} \\
A+B \mathcal{H}_{c} L & A \mathcal{R}+B \mathcal{G}_{c}
\end{array}\right], \\
& \Gamma_{P}^{T} \tilde{B}_{0} \Gamma_{S} \triangleq\left[\begin{array}{cc}
\mathcal{P} B_{0}+\mathcal{B}_{c} F_{0} & \mathcal{B}_{0 c} \\
B_{0}+B \mathcal{H}_{c} F_{0} & B_{0} \mathcal{S}+B \mathcal{H}_{0 c}
\end{array}\right], \\
& \Gamma_{Q}^{T} \tilde{C} \Gamma_{R} \triangleq\left[\begin{array}{cc}
\mathcal{Q} C+\mathcal{D}_{c} L & \mathcal{C}_{c} \\
C+D \mathcal{H}_{c} L & C \mathcal{R}+D \mathcal{G}_{c}
\end{array}\right], \\
& \Gamma_{Q}^{T} \tilde{D}_{0} \Gamma_{S} \triangleq\left[\begin{array}{cc}
\mathcal{Q} D_{0}+\mathcal{D}_{c} F_{0} & \mathcal{D}_{0 c} \\
D_{0}+D \mathcal{H}_{c} F_{0} & D_{0} \mathcal{S}+D \mathcal{H}_{0 c}
\end{array}\right] \text {, } \\
& \Gamma_{P}^{T} \tilde{B}_{1} \triangleq\left[\begin{array}{c}
\mathcal{P} B_{1}+\mathcal{B}_{c} F_{1} \\
B_{1}+B \mathcal{H}_{c} F_{1}
\end{array}\right], \Gamma_{P}^{T} \tilde{M}_{1} \triangleq\left[\begin{array}{c}
\mathcal{P} M_{1} \\
M_{1}
\end{array}\right], \\
& \Gamma_{Q}^{T} \tilde{D}_{1} \triangleq\left[\begin{array}{c}
\mathcal{Q} D_{1}+\mathcal{D}_{c} F_{1} \\
D_{1}+D \mathcal{H}_{c} F_{1}
\end{array}\right], \Gamma_{Q}^{T} \tilde{M}_{2} \triangleq\left[\begin{array}{c}
\mathcal{Q} M_{2} \\
M_{2}
\end{array}\right] \text {, } \\
& \Gamma_{P}^{T} \Gamma_{R} \triangleq\left[\begin{array}{cc}
\mathcal{P} & I \\
I & \mathcal{R}
\end{array}\right], \Gamma_{Q}^{T} \Gamma_{S} \triangleq\left[\begin{array}{cc}
\mathcal{Q} & I \\
I & \mathcal{S}
\end{array}\right], \\
& \tilde{G} \Gamma_{R} \triangleq\left[\begin{array}{ll}
G & G \mathcal{R}
\end{array}\right], \quad \tilde{H}_{0} \Gamma_{S} \triangleq\left[\begin{array}{ll}
H_{0} & H_{0} \mathcal{S}
\end{array}\right], \\
& \tilde{N}_{1} \Gamma_{R} \triangleq\left[\begin{array}{ll}
N_{1}+N_{3} \mathcal{H}_{c} L & N_{1} \mathcal{R}+N_{3} \mathcal{G}_{c}
\end{array}\right], \\
& \tilde{N}_{2} \Gamma_{S} \triangleq\left[\begin{array}{ll}
N_{2}+N_{3} \mathcal{H}_{c} F_{0} & N_{2} \mathcal{S}+N_{3} \mathcal{H}_{0 c}
\end{array}\right] \text {. }
\end{aligned}
$$

Substituting (54) into (51) and (52), we obtain

$$
\left[\begin{array}{ccccccc}
\Psi_{11} & \Psi_{12} & \Psi_{13} & \mathcal{B}_{0 c} & \Psi_{15} & \Psi_{16} & \Psi_{17} \\
* & \Psi_{22} & \Psi_{23} & \Psi_{24} & \Psi_{25} & \mathcal{C}_{c}^{T} & \Psi_{27} \\
* & * & -\mathcal{Q} & -I & 0 & \Psi_{36} & \Psi_{37} \\
* & * & * & -\mathcal{S} & 0 & \mathcal{D}_{0 c}^{T} & \Psi_{47} \\
* & * & * & * & -I & \Psi_{56} & \Psi_{57} \\
* & * & * & * & * & -\mathcal{Q} & -I \\
* & * & * & * & * & * & -\mathcal{S}
\end{array}\right]<0
$$

and (44), respectively, where $\Psi_{11}, \Psi_{12}, \Psi_{13}, \Psi_{22}, \Psi_{23}, \Psi_{24}, \Psi_{15}, \Psi_{25}, \Psi_{16}, \Psi_{36}, \Psi_{56}, \Psi_{17}, \Psi_{27}, \Psi_{37}, \Psi_{47}$ and $\Psi_{57}$ are defined in (45). Now, consider the parameter uncertainties, that is, replace $A, B_{0}, C$ and $D_{0}$ with 
$(A+\Delta A),\left(B_{0}+\Delta B_{0}\right),(C+\Delta C)$ and $\left(D_{0}+\Delta D_{0}\right)$ in $(55)$, respectively. It can be seen that the new inequality (55) holds if the following holds:

$$
\left[\begin{array}{ccccccc}
\Psi_{11} & \Psi_{12} & \Psi_{13} & \mathcal{B}_{0 c} & \Psi_{15} & \Psi_{16} & \Psi_{17} \\
* & \Psi_{22} & \Psi_{23} & \Psi_{24} & \Psi_{25} & \mathcal{C}_{c}^{T} & \Psi_{27} \\
* & * & -\mathcal{Q} & -I & 0 & \Psi_{36} & \Psi_{37} \\
* & * & * & -\mathcal{S} & 0 & \mathcal{D}_{0 c}^{T} & \Psi_{47} \\
* & * & * & * & -I & \Psi_{56} & \Psi_{57} \\
* & * & * & * & * & -\mathcal{Q} & -I \\
* & * & * & * & * & * & -\mathcal{S}
\end{array}\right]+\operatorname{sym}\left(\left[\begin{array}{c}
0 \\
M_{1} \\
0 \\
0 \\
0 \\
0 \\
M_{2}
\end{array}\right] \Delta\left[\begin{array}{c}
\Psi_{18} \\
\Psi_{28} \\
\Psi_{38} \\
\Psi_{48} \\
\Psi_{58} \\
0 \\
0
\end{array}\right]^{T}+\left[\begin{array}{c}
\mathcal{P} M_{1} \\
M_{1} \\
0 \\
0 \\
0 \\
\mathcal{Q} M_{2} \\
M_{2}
\end{array}\right] \Delta\left[\begin{array}{c}
N_{1}^{T} \\
0 \\
N_{2}^{T} \\
0 \\
0 \\
0 \\
0
\end{array}\right]^{T}\right)
$$

where $\Psi_{18}, \Psi_{28}, \Psi_{38}, \Psi_{48}$ and $\Psi_{58}$ are defined in (45). By invoking Lemma 1 together with Schur complement, (56) holds if (43) holds.

On the other hand, substituting $\mathcal{P} \triangleq P_{11}, \mathcal{R} \triangleq R_{11}, \mathcal{Q} \triangleq Q_{11}$ and $\mathcal{S} \triangleq S_{11}$ into (53) supplies (46). Therefore, we can conclude from Theorem 3 that the uncertain closed-loop LRP $\tilde{\Sigma}_{D}$ is robustly stable along the pass with an $L_{2}-L_{\infty}$ performance level $\gamma_{2, \infty}$. The proof is complete.

Remark 1: It should be pointed out that, in order to obtain the parameters of output feedback controller in (46), matrices $P_{12}, R_{12}, Q_{12}$ and $S_{12}$, which should be available in advance, can be obtained by taking any full rank factorization of $P_{12} R_{12}^{T}=I-\mathcal{P} \mathcal{R}$ and $Q_{12} S_{12}^{T}=I-\mathcal{Q S}$, respectively (derived from $P_{11} R_{11}+P_{12} R_{12}^{T}=I$ and $Q_{11} S_{11}+Q_{12} S_{12}^{T}=I$, respectively).

Remark 2: Note that Theorem 4 provides a sufficient condition for the solvability of $L_{2}$ - $L_{\infty}$ dynamic output feedback control problem for the differential LRP. Since the obtained condition is of the LMI form, a desired controller can be determined by solving the following convex optimization problem:

$$
\min \delta \quad \text { subject to }(43)-(44) \quad\left(\text { where } \delta=\gamma_{2, \infty}^{2}\right)
$$

\section{An Illustrative Example}

Consider the differential LRP $\Sigma$ in (1) with $\alpha=20, k \geq 0$, with system matrices given as follows:

$$
\begin{aligned}
& A=\left[\begin{array}{rrr}
0.1 & 0.4 & 0.0 \\
0.0 & -0.9 & 0.1 \\
0.8 & 0.0 & -1.2
\end{array}\right], B_{0}=\left[\begin{array}{rr}
-0.5 & -0.1 \\
-0.1 & -0.1 \\
0.5 & 0.4
\end{array}\right], B=\left[\begin{array}{l}
0.1 \\
0.1 \\
0.1
\end{array}\right], B_{1}=\left[\begin{array}{l}
0.1 \\
0.0 \\
0.1
\end{array}\right] \\
& C=\left[\begin{array}{lll}
0.4 & 0.2 & 0.3 \\
0.5 & 0.4 & 0.6
\end{array}\right], D_{0}=\left[\begin{array}{ll}
0.6 & 0.3 \\
0.2 & 0.5
\end{array}\right], D=\left[\begin{array}{l}
0.4 \\
0.5
\end{array}\right], D_{1}=\left[\begin{array}{l}
0.1 \\
0.2
\end{array}\right] \\
& L=\left[\begin{array}{lll}
0.2 & 0.2 & 0.1 \\
0.0 & 0.3 & 0.1
\end{array}\right], F_{0}=\left[\begin{array}{ll}
0.1 & 0.2 \\
0.0 & 0.1
\end{array}\right], F_{1}=\left[\begin{array}{l}
0.3 \\
0.2
\end{array}\right], H_{0}=\left[\begin{array}{ll}
0.1 & 0.3 \\
0.2 & 0.1
\end{array}\right] \\
& M_{1}=\left[\begin{array}{lll}
0.2 & 0.1 & 0.1 \\
0.1 & 0.2 & 0.0 \\
0.0 & 0.1 & 0.1
\end{array}\right], N_{1}=\left[\begin{array}{lll}
0.1 & 0.2 & 0.0 \\
0.1 & 0.0 & 0.1 \\
0.2 & 0.0 & 0.1
\end{array}\right], N_{2}=\left[\begin{array}{ll}
0.1 & 0.0 \\
0.1 & 0.1 \\
0.0 & 0.2
\end{array}\right], N_{3}=\left[\begin{array}{l}
0.1 \\
0.1 \\
0.2
\end{array}\right] \\
& M_{2}=\left[\begin{array}{lll}
0.2 & 0.0 & 0.1 \\
0.0 & 0.1 & 0.2
\end{array}\right], G=\left[\begin{array}{lll}
0.2 & 0.2 & 0.1 \\
0.3 & 0.1 & 0.4
\end{array}\right]
\end{aligned}
$$

and $\Delta(t)=\operatorname{diag}(\sin t, \cos t, \sin t)$. According to Lemma 1 , the unforced process $\Sigma$ in (1) with above matrices is unstable along the pass. Our attention is to design an $L_{2}-L_{\infty}$ static feedback controller with the form of (4) and an $L_{2}-L_{\infty}$ dynamic output feedback controller with the form of (10), such that the corresponding closed-loop LRPs are robustly stable along the pass and have an $L_{2}-L_{\infty}$ performance level $\gamma_{2, \infty}$. 
First, we deal with the $L_{2}-L_{\infty}$ static feedback control problem. Solving LMIs (35)-(36) in Theorem 2, we obtain the achieved minimum $\gamma_{2, \infty}^{\#}=0.2246$ and $\lambda_{1}=0.2405, \lambda_{2}=0.0066$ and

$$
\begin{aligned}
& \mathcal{P}_{1}=\left[\begin{array}{rrr}
0.5403 & -0.0468 & 0.2607 \\
-0.0468 & 0.0744 & -0.0080 \\
0.2607 & -0.0080 & 0.1945
\end{array}\right], \quad \mathcal{P}_{2}=\left[\begin{array}{rr}
0.0588 & -0.0174 \\
-0.0174 & 0.2203
\end{array}\right] \\
& \mathcal{X}=\left[\begin{array}{llr}
-0.7864 & 0.0023 & -0.4634
\end{array}\right], \quad \mathcal{Y}=\left[\begin{array}{ll}
-0.0258 & -0.1919
\end{array}\right]
\end{aligned}
$$

Thus, the parameter matrices in (4) are computed as

$$
\begin{aligned}
& K_{1}=\mathcal{X} \mathcal{P}_{1}^{-1}=\left[\begin{array}{lll}
-1.0013 & -0.7137 & -1.0696
\end{array}\right] \\
& K_{2}=\mathcal{Y P}_{2}^{-1}=\left[\begin{array}{ll}
-0.7144 & -0.9275
\end{array}\right]
\end{aligned}
$$

Next, we consider the $L_{2}-L_{\infty}$ dynamic output feedback control problem. Solving the LMI conditions (43)(44) in Theorem 4 and choosing $R_{12}=I$ and $S_{12}=I$, we obtain that the achieved minimum $\gamma_{2, \infty}$ is $\gamma_{2, \infty}^{*}=$ 0.8353 and the associate parameters for the desired $L_{2}-L_{\infty}$ dynamic output feedback controller are given by

$$
\begin{aligned}
& A_{c}=\left[\begin{array}{rrr}
-9.5543 & 4.7168 & -3.9763 \\
-9.3027 & 1.5701 & -5.4632 \\
3.3674 & -4.2751 & -0.6981
\end{array}\right], B_{0 c}=\left[\begin{array}{rr}
-0.3270 & -1.7050 \\
0.2366 & -1.1796 \\
0.6321 & 1.9241
\end{array}\right] \\
& B_{c}=\left[\begin{array}{rr}
19.8034 & -33.6044 \\
23.3787 & -31.3793 \\
-2.2520 & 13.8605
\end{array}\right], C_{c}=\left[\begin{array}{rrr}
-1.3702 & 0.1725 & -0.7217 \\
-0.0190 & -0.1200 & -0.0062
\end{array}\right] \\
& D_{0 c}=\left[\begin{array}{rr}
0.0988 & -0.3295 \\
-0.0478 & 0.0839
\end{array}\right], D_{c}=\left[\begin{array}{rr}
2.3986 & -3.5164 \\
-0.1259 & 0.3775
\end{array}\right] \\
& G_{c}=\left[\begin{array}{lll}
1.9111 & -1.6333 & 0.9006
\end{array}\right], H_{0 c}=\left[\begin{array}{ll}
0.0952 & -0.2815
\end{array}\right] \\
& H_{c}=\left[\begin{array}{ll}
-11.2830 & 15.5137
\end{array}\right]
\end{aligned}
$$

To show the state responses of the open-loop and closed-loop processes, suppose that the boundary conditions are given as

$$
\begin{array}{rll}
x_{k+1}(0) & =0.2, & 0 \leq k \leq 20 \\
y_{0}(t) & =0.2, & 0 \leq t \leq 20
\end{array}
$$

Figure 1 shows the state response of the first state component of the open-loop process from which we can see that it is not stable along the pass. Figure 2 gives the corresponding result for the closed-loop process (use the static $L_{2}-L_{\infty}$ control).

\section{Concluding Remarks}

This paper has investigated the robust $L_{2}-L_{\infty}$ control problem for uncertain differential LRPs, which are a distinct class of two-dimensional (2-D) linear systems. Both the $L_{2}-L_{\infty}$ static feedback controller and the $L_{2}-L_{\infty}$ dynamic output feedback controller have been designed with the solvability conditions proposed in terms of LMI respectively. The designed $L_{2}-L_{\infty}$ dynamic output feedback controller can be found by solving a convex optimization problem. A numerical example has been provided to demonstrate the effectiveness of the proposed design method. Note that the LMI formulation in this paper is mainly based on a fixed Lyapunov functional. To further reduce the possible conservatism, one of our future research topics would be the investigation on linear repetitive processes with polytopic parameter uncertainties by exploiting parameterdependent Lyapunov functionals. 


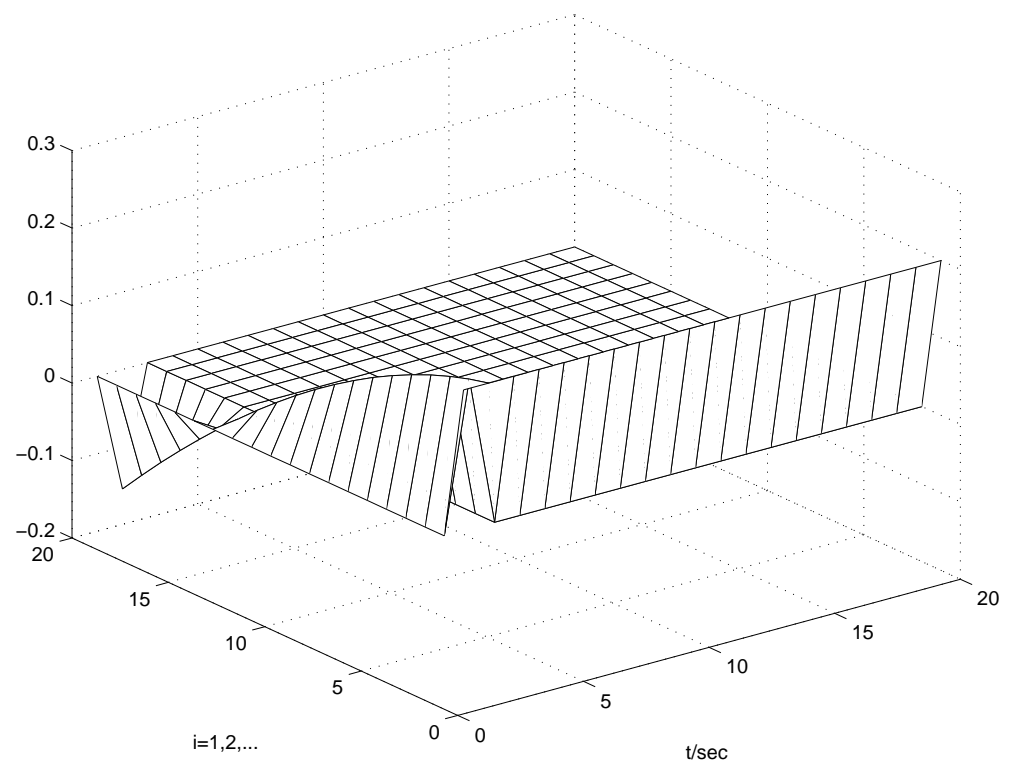

Fig. 1. State response of the first state component of the open-loop process

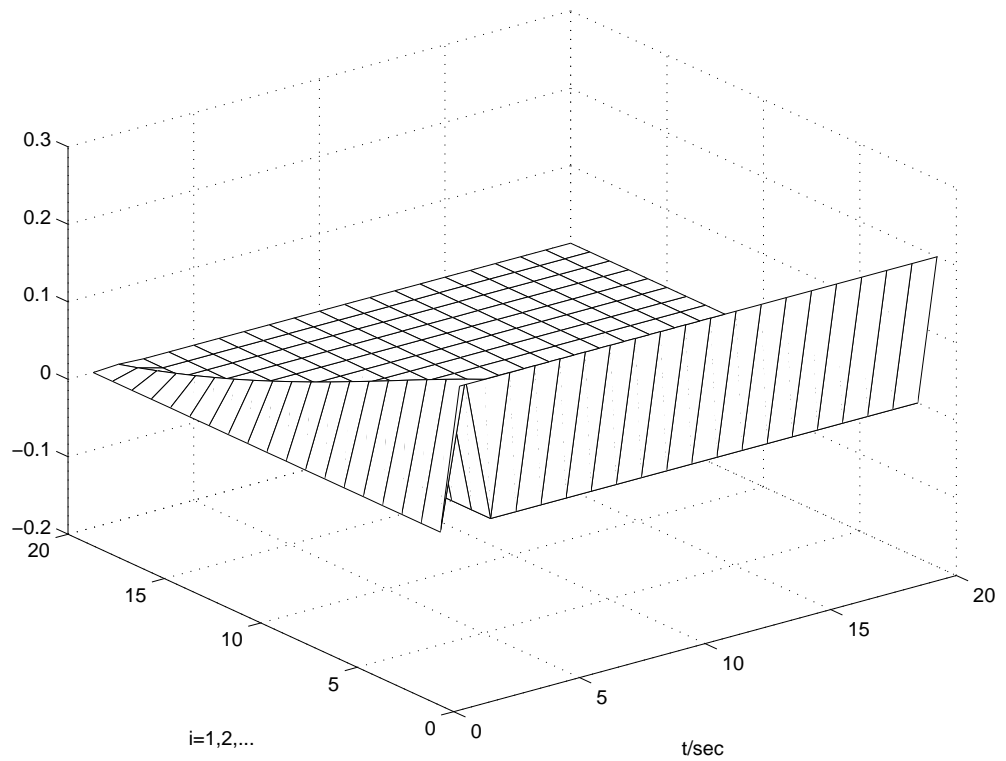

Fig. 2. State response of the first state component of the closed-loop process

\section{REFERENCES}

[1] C. Du and L. Xie. $H_{\infty}$ control and Filtering of Two-dimensional Systems, volume 278 of Lecture Notes in Control and Information Sciences. Springer-Verlag, Berlin, Germany, 2002.

[2] P. Gahinet, A. Nemirowski, A. J. Laub, and M. Chilali. LMI Control Toolbox for Use with MATLAB. The Mathworks Partner Series, The MathWorks Inc., 1995.

[3] K. Galkowski, W. Paszke, E. Rogers, S. Xu, J. Lam, and D. H. Owens. Stability and control of differential linear repetitive processes using an LMI setting. IEEE Trans. Circuits and Systems II, 50(9):662-666, 2003.

[4] H. Gao, C. Wang, and J. Wang. On $H_{\infty}$ performance analysis for continuous-time stochastic systems with polytopic uncertainties. Circuits, Systems and Signal Processing, 24(4):415-429, 2005.

[5] H. Gao, J. Lam, C. Wang and S. Xu. $H_{\infty}$ model reduction for uncertain two-dimensional discrete systems. Optimal Control Applications \&3 Methods. 26(4): 199-227, 2005. 
[6] H. Gao, J. Lam and C. Wang, Model simplification for switched hybrid systems, Systems E Control Letters, 55(12): 1015$1021,2006$.

[7] H. Gao, J. Lam and C. Wang, Robust energy-to-peak filter design for stochastic time-delay systems, Systems E Control Letters, 55(2): 101-111, 2006.

[8] E. N. Goncalves, R. M. Palhares, and R. H. C. Takahashi. Improved optimisation approach to the robust $H_{2} / H_{\infty}$ control problem for linear systems, IEE Proc. Part D: Control Theory Appl., 152(2): 171-176, 2005.

[9] E. N. Goncalves, R. M. Palhares, and R. H. C. Takahashi. $H_{2} / H_{\infty}$ Filter Design for Systems With Polytope-Bounded Uncertainty, IEEE Trans. Signal Processing, 54(9): 3620-3626, 2006.

[10] S. Kanev, C. Scherer, M. Verhaegen, and B. De Schutter. Robust output-feedback controller design via local BMI optimization. Automatica, 40(7):1115-1127, 2004.

[11] P. P. Khargonekar, I. R. Petersen, and K. Zhou. Robust stabilization of uncertain linear systems: quadratic stabilizability and $H_{\infty}$ control theory. IEEE Trans. Automat. Control, 35(3):356-361, 1990.

[12] W. S. Lu and A. Antoniou. Two-Dimensional Digital Filters. Marcel Dekker, New York, 1992.

[13] R. M. Palhares and P. L. D. Peres. Robust filtering with guaranteed energy-to-peak performance: an LMI approach. Automatica, 36(6):851-858, 2000.

[14] W. Paszke, K. Galkowski, E. Rogers, and D. H. Owens. Guaranteed cost control of uncertain differential linear repetitive processes. IEEE Trans. Circuits and Systems II, 51(11):629-634, 2004.

[15] W. Paszke, K. Galkowski, E. Rogers, and D. H. Owens. $H_{\infty}$ control of differential linear repetitive processes. IEEE Trans. Circuits and Systems I, 53(1):39-44, 2006.

[16] W. Paszke, K. Galkowski, E. Rogers, and D. H. Owens. $H_{\infty}$ and guaranteed cost control of discrete linear repetitive processes. Linear Algebra and its Application, 412(2-3):93-131, 2006.

[17] E. Rogers and D. H. Owens. Stability Analysis for Linear Repetitive Processes, volume 175 of Lecture Notes in Control and Information Sciences. Springer-Verlag, Berlin, Germany, 1992.

[18] C. W. Scherer. Mixed $H_{2} / H_{\infty}$ control, A. Isidori, Ed., Trends in Control, A European Perspective. Springer-Verlag, Berlin, Germany, 1995.

[19] P. Shi, R. K. Agarwal, E. K. Boukas, and S. P. Shue. Robust $H_{\infty}$ state feedback control of discrete time-delay linear systems with norm-bounded uncertainty, Int. J. Systems Science, 31(4): 409-415, 2000.

[20] P. Shi and S. K. Nguang. $H_{\infty}$ output feedback control of fuzzy system models under sampled measurements, Computers $\mathcal{E}_{6}$ Mathematics with Applications, 46(5-6): 705-717, 2003.

[21] K. Tan, K. M. Grigoriadis, and F. Wu. $H_{\infty}$ and $L_{2}-L_{\infty}$ gain control of linear parameter-varying systems with parametervarying delays. IEE Proc. Part D: Control Theory Appl., 150(5):509-517, 2003.

[22] Z. Wang, F. Yang, D. W. C. Ho and X. Liu. Robust $H_{\infty}$ filtering for stochastic time-delay systems with missing measurements. IEEE Transactions on Signal Processing, 54(7): 2579-2587, 2006.

[23] Z. Wang and D. W. C. Ho. Output feedback robust $H_{\infty}$ control with D-stability and variance constraints: a parameterization approach. Journal of Dynamical and Control Systems, 11(2): 263-280, 2005.

[24] L. Xie, C. Du, Y. C. Soh, and C. Zhang. $H_{\infty}$ and robust control of 2-D systems in FM second model. Multidimensional Systems and Signal Processing, 13:256-287, 2002.

[25] F. Yang, Z. Wang and D. W. C. Ho. Robust mixed $H_{2} / H_{\infty}$ control for a class of nonlinear stochastic systems. IEE Proceedings - Control Theory and Applications, 153(2): 175-184, 2006.

[26] L. Zhang, B. Huang and L. Lam. $H_{\infty}$ model reduction of Markovian jump linear systems. Systems 8 Control Letters, 50(2): $103-118,2003$ 